\title{
Sommaire
}

Avant-propos : par Paul Rigny ......................... 9

Préface : par Bernard Bigot........................... 11

Introduction : par Armand Lattes .................. $\quad 15$

Partie 1

La chimie pour comprendre la nature

Chapitre 1 : La chimie, un outil pour comprendre la nature

par Michel Rohmer.....

Chapitre 2 : La chimie atmosphérique : contexte, récents développements et applications

par Éric Villenave

Chapitre 3 : Biogéochimie et écologie des sols par Jean-François Soussana

Chapitre 4 : L'eau, sa purification et les micropolluants

par Marina Coquery et Samuel Martin Ruel

\author{
Partie 2 \\ La nature pour inspirer la chimie
}

Chapitre 5: La nature pour inspirer le chimiste : substances naturelles, phytochimie et chimie médicinale

par Françoise Guéritte

Chapitre 6 : Matériaux inorganiques et hybrides bio-inspirés

par Clément Sanchez

Chapitre 7 : À la frontière de la chimie et de la biologie : biocatalyse et catalyse bio-inspirée 


\section{Partie 3}

La chimie pour protéger la nature

et ses ressources

Chapitre 8 : Phytoremédiation des sols contaminés : des plantes pour guérir les sols par Jean-Louis Morel

Chapitre 9 : De la chimie des écosystèmes et des cocktails...

par Éric Blin

Chapitre 10 : Le dioxyde de carbone, la moléculeclé de la chimie du développement durable par Jacques Amouroux, Paul Siffert, JeanPierre Massué, Simeon Cavadias, Béatriz Trujillo, Koshi Hashimoto, Phillip Rutberg et Sergey Dresvin

\section{Partie 4 \\ La chimie pour mieux utiliser les ressources naturelles}

Chapitre 11 : Recyclage des métaux : mimer les processus naturels par Bruno Goffé

Chapitre 12 : Valorisation biologique des agroressources

par Pierre Monsan

Chapitre 13 : Chimie du végétal, fer de lance de la chimie durable

D'après la conférence de Christophe RuppDahlem 